\title{
Hand Digit 1 Phalanx
}

National Cancer Institute

\section{Source}

National Cancer Institute. Hand Digit 1 Phalanx. NCI Thesaurus. Code C52777.

A bone in the thick, short finger next to the index finger. 